\title{
Fuzzy b-Metric Spaces
}

\author{
S. Nădăban
}

\author{
Sorin Nădăban \\ Department of Mathematics and Computer Science \\ Aurel Vlaicu University of Arad, \\ Elena Drăgoi 2, RO-310330 Arad, Romania \\ snadaban@gmail.com
}

\begin{abstract}
Metric spaces and their various generalizations occur frequently in computer science applications. This is the reason why, in this paper, we introduced and studied the concept of fuzzy b-metric space, generalizing, in this way, both the notion of fuzzy metric space introduced by I. Kramosil and J. Michálek and the concept of b-metric space. On the other hand, we introduced the concept of fuzzy quasi-bmetric space, extending the notion of fuzzy quasi metric space recently introduced by V. Gregori and S. Romaguera. Finally, a decomposition theorem for a fuzzy quasipseudo-b-metric into an ascending family of quasi-pseudo-b-metrics is established. The use of fuzzy b-metric spaces and fuzzy quasi-b-metric spaces in the study of denotational semantics and their applications in control theory will be an important next step.
\end{abstract}

Keywords: Fuzzy b-metric spaces, fuzzy quasi-b-metric, fuzzy quasi-pseudo-bmetric, b-metric space.

\section{Introduction and preliminaries}

The concept of b-metric space was introduced by I.A. Bakhtin [5] and exensively used by S. Czerwic $[10,11]$.

Definition 1. [10] Let $X$ be a nonempty set and $k \geq 1$ be a given real number. A function $d: X \times X \rightarrow[0, \infty)$ is a b-metric on $X$ if, for all $x, y, z \in X$, the following conditions hold:

(b1) $d(x, y)=0$ if and only if $x=y$;

(b2) $d(x, y)=d(y, x)$;

(b3) $d(x, z) \leq k[d(x, y)+d(y, z)]$.

The triple $(X, d, k)$ will be called b-metric space.

Some examples of b-metric spaces and some fixed point theorems in b-metric spaces can be found in $[6-8,21]$. We also note that the class of b-metric spaces is larger than that of metric spaces, since every b-metric is a metric when $k=1$. In [22] an example of a b-metric space which is not a metric space, is given.

Recently, M.A. Alghamdi, N. Hussain, P. Salimi [1] introduced the notion of b-metric-like space, which is an interesting generalization of metric-like space (introduced by A. Amini-Harandi [2]) and partial metric space (introduced by S.G. Matthews [17]). In paper [14], N. Hussain and M.H. Shah introduced the notion of cone b-metric space, generalizing both notions of b-metric spaces and cone metric spaces.

The concept of quasi-b-metric space was introduced by M.H. Shah and N. Hussain [20] in 2012. In this paper we adopt a slight modification of their definition.

Definition 2. Let $X$ be a nonempty set. A real valued function $d: X \times X \rightarrow[0, \infty)$ is said to be a quasi-b-metric with constant $k \geq 1$ if the following conditions are satisfied: 
(qb1) $d(x, y)=d(y, x)=0$ if and only if $x=y$;

(qb3) $d(x, z) \leq k[d(x, y)+d(y, z)],(\forall) x, y, z \in X$.

The triple $(X, d, k)$ will be called quasi-b-metric space.

On the other hand, after L.A. Zadeh has introduced in his famous paper [23] the concept of fuzzy set, one of the important problems is to obtain an adequate notion of fuzzy metric space. I. Kramosil and J. Michálek [16] reformulated successfully the notion of probabilistic metric space, introduced by K. Menger in 1942, in fuzzy context.

Definition 3. [19] A binary operation

$$
*:[0,1] \times[0,1] \rightarrow[0,1]
$$

is called triangular norm (t-norm) if it satisfies the following condition:

1. $a * b=b * a,(\forall) a, b \in[0,1]$;

2. $a * 1=a,(\forall) a \in[0,1]$;

3. $(a * b) * c=a *(b * c),(\forall) a, b, c \in[0,1]$;

4. If $a \leq c$ and $b \leq d$, with $a, b, c, d \in[0,1]$, then $a * b \leq c * d$.

Example 4. Three basic examples of continuous t-norms are $\wedge, \cdot, *_{L}$, which are defined by $a \wedge b=$ $\min \{a, b\}, a \cdot b=a b$ (usual multiplication in $[0,1]$ ) and $a *_{L} b=\max \{a+b-1,0\}$ (the Lukasiewicz t-norm).

Definition 5. [16] The triple $(X, M, *)$ is said to be a fuzzy metric space if $X$ is an arbitrary set, * is a continuous t-norm and $M$ is a fuzzy metric, i.e. a fuzzy set in $X \times X \times[0, \infty)$ such that for all $x, y, z \in X$ we have:

(M1) $M(x, y, 0)=0$;

(M2) $[M(x, y, t)=1,(\forall) t>0]$ if and only if $x=y$;

(M3) $M(x, y, t)=M(y, x, t),(\forall) t \geq 0$;

(M4) $M(x, z, t+s) \geq M(x, y, t) * M(y, z, s),(\forall) t, s \geq 0$;

(M5) $M(x, y, \cdot):[0, \infty) \rightarrow[0,1]$ is left continuous and $\lim _{t \rightarrow \infty} M(x, y, t)=1$.

We note that A. George and P. Veeramani [12] modified the concept of fuzzy metric space introduced by I. Kramosil and J. Michálek and defined a Hausdorff topology on this fuzzy space. Another approach for fuzzy metric spaces was introduced by O. Kaleva and S. Seikkala in paper [15], by setting the distance between two points to be a non-negative, upper semicontinuous, normal and convex fuzzy number.

In recent years, different types of fuzzy generalized metric spaces was considered by different authors in different approaches. Thus, V. Gregori and S. Romaguera introduced in paper [13] the concept of fuzzy quasi-metric space, generalizing in this way the notions of fuzzy metric introduced by I. Kramosil and J. Michálek and by A. George and P. Veeramani to the quasimetric setting.

On the other hand, the idea of fuzzy cone metric space has been introduced in [3] and some basic properties and fixed point theorems for different types of contraction mappings have been developed in fuzzy cone metric spaces. In paper [4], T. Bag introduced the concept of fuzzy 
cone b-metric space and some fixed point theorems are established in such spaces for contraction mappings. We must note that Bag's definitions for fuzzy cone metric space and for fuzzy cone b-metric spaces generalized the notion of fuzzy metric introduced by Kaleva and Seikkala.

In this paper we introduced and studied the concept of fuzzy b-metric space, generalizing, in this way, both the notion of fuzzy metric space introduced by I. Kramosil and J. Michálek and the concept of b-metric space. On the other hand, we introduced the concept of fuzzy quasi-bmetric space, extending the notion of fuzzy quasi-metric space recently introduced by V. Gregori and S. Romaguera. Finally, a decomposition theorem for a fuzzy quasi-pseudo-b-metric into an ascending family of quasi-pseudo-b-metrics is established.

\section{$2 \quad$ Fuzzy b-metric spaces}

Definition 6 . Let $X$ be a nonempty set, let $k \geq 1$ be a given real number and $*$ be a continuous t-norm. A fuzzy set $M$ in $X \times X \times[0, \infty)$ is called fuzzy b-metric if, for all $x, y, z \in X$, the following conditions hold:

(bM1) $M(x, y, 0)=0$;

(bM2) $[M(x, y, t)=1,(\forall) t>0]$ if and only if $x=y$;

(bM3) $M(x, y, t)=M(y, x, t),(\forall) t \geq 0$;

(bM4) $M(x, z, k(t+s)) \geq M(x, y, t) * M(y, z, s),(\forall) t, s \geq 0$;

(bM5) $M(x, y, \cdot):[0, \infty) \rightarrow[0,1]$ is left continuous and $\lim _{t \rightarrow \infty} M(x, y, t)=1$.

The quadruple $(X, M, *, k)$ is said to be a fuzzy b-metric space.

Remark 7. The class of fuzzy b-metric spaces is larger than the class of fuzzy metric spaces, since a fuzzy b-metric space is a fuzzy metric space when $k=1$.

Example 8. Let $(X, d, k)$ be a b-metric space. Let

$$
M_{d}: X \times X \times[0, \infty) \rightarrow[0,1], M_{d}(x, y, t)=\left\{\begin{array}{rll}
\frac{t}{t+d(x, y)} & \text { if } t>0 \\
0 & \text { if } t=0
\end{array} .\right.
$$

Then $\left(X, M_{d}, \wedge, k\right)$ is a fuzzy b-metric space. $M_{d}$ will be called standard fuzzy b-metric.

Proof: We check only (bM4), because verifying the other conditions is standard.

Let $x, y, z \in X$ and $t, s>0$. Without restraining the generality we assume that $M_{d}(x, y, t) \leq$ $M_{d}(y, z, s)$. Thus $\frac{t}{t+d(x, y)} \leq \frac{s}{s+d(y, z)}$, i.e. $t d(y, z) \leq s d(x, y)$.

On the other hand

$$
\begin{gathered}
M_{d}(x, z, k(t+s))=\frac{k(t+s)}{k(t+s)+d(x, z)} \geq \\
\geq \frac{k(t+s)}{k(t+s)+k[d(x, y)+d(y, z)]}=\frac{t+s}{t+s+d(x, y)+d(y, z)} .
\end{gathered}
$$

We will prove that

$$
\frac{t+s}{t+s+d(x, y)+d(y, z)} \geq \frac{t}{t+d(x, y)} .
$$

Hence we will obtain that $M_{d}(x, z, k(t+s)) \geq M_{d}(x, y, t)=M_{d}(x, y, t) \wedge M_{d}(y, z, s)$, what had to be verified. We remark that

$$
\frac{t+s}{t+s+d(x, y)+d(y, z)} \geq \frac{t}{t+d(x, y)} \Leftrightarrow
$$




$$
t^{2}+s t+t d(x, y)+s d(x, y) \geq t^{2}+s t+t d(x, y)+t d(y, z) \Leftrightarrow s d(x, y) \geq t d(y, z),
$$

which is true.

Definition 9 . Let $k \geq 1$ be a real given number. A function $f: \mathbb{R} \rightarrow \mathbb{R}$ will be called $k$ nondecreasing if for $t<s$ we will have that $f(t) \leq f(k s)$.

Proposition 10. For all $x, y \in X$ the mapping $M(x, y, \cdot):[0, \infty) \rightarrow[0,1]$ is k-nondecreasing.

Proof: For $0<t<s$ we have

$$
M(x, y, k s) \geq M(x, x, s-t) * M(x, y, t)=1 * M(x, y, t)=M(x, y, t) .
$$

Theorem 2.1. Let $(X, M, *, k)$ be a fuzzy b-metric space. For $x \in X, r \in(0,1)$, $t>0$ we define the open ball

$$
B(x, r, t):=\{y \in X: M(x, y, t)>1-r\} .
$$

Then

$$
\mathcal{T}_{M}:=\{T \subset X: x \in T \text { iff }(\exists) t>0, r \in(0,1): B(x, r, t) \subseteq T\}
$$

is a topology on $X$.

Proof: It is obvious that $\emptyset$ and $X$ belong to $\mathcal{T}_{M}$.

Let $\left\{T_{i}\right\}_{i \in I} \subseteq \mathcal{T}_{M}$ and $T=\bigcup_{i \in I} T_{i}$. We will show that $T \in \mathcal{T}_{M}$. Let $x \in T$. Then there exists $i_{0} \in I$ such that $x \in T_{i_{0}}$. As $T_{i_{0}} \in \mathcal{T}_{M}$, there exist $t>0, r \in(0,1)$ such that $B(x, r, t) \subseteq T_{i_{0}}$. Thus $B(x, r, t) \subseteq \bigcup_{i \in I} T_{i}=T$.

Let now $\left\{T_{i}\right\}_{i=1}^{n} \subseteq \mathcal{T}_{M}$ and $T=\bigcap_{i=1}^{n} T_{i}$. We will show that $T \in \mathcal{T}_{M}$. Let $x \in T$. We obtain that $x \in T_{i},(\forall) i=\overline{1, n}$. Thus

$$
(\exists) t_{i}>0, r_{i} \in(0,1): B\left(x, r_{i}, t_{i}\right) \subseteq T_{i},(\forall) i=\overline{1, n} .
$$

Let

$$
r=\min \left\{r_{i}, i=\overline{1, n}\right\}, t=\min \left\{\frac{t_{i}}{k}, i=\overline{1, n}\right\} .
$$

We have that $B(x, r, t) \subseteq B\left(x, r_{i}, t_{i}\right),(\forall) i=\overline{1, n}$. Indeed, for $y \in B(x, r, t)$, we have $M(x, y, t)>1-r \geq 1-r_{i},(\forall) i=\overline{1, n}$. As $t \leq \frac{t_{i}}{k},(\forall) i=\overline{1, n}$, we obtain that $M(x, y, t) \leq M\left(x, y, t_{i}\right)$. Thus $M\left(x, y, t_{i}\right)>1-r_{i},(\forall) i=\overline{1, n}$. Hence $y \in B\left(x, r_{i}, t_{i}\right),(\forall) i=\overline{1, n}$. Therefore $B(x, r, t) \subseteq T_{i},(\forall) i=\overline{1, n}$. Thus $B(x, r, t) \subseteq \bigcap_{i=1}^{n} T_{i}=T$.

Remark 11. Previous theorem extends to fuzzy b-metric space a similar result obtained by A. George and P. Veeramani [12] in the context of fuzzy metric space. The definitions for convergent sequence and Cauchy sequence given by A. George and P. Veeramani [12] in the context of fuzzy metric space can be translated in the context of fuzzy b-metric space, as follows.

Definition 12 . Let $(X, M, *, k)$ be a fuzzy b-metric space and $\left(x_{n}\right)$ be a sequence in $X$. The sequence $\left(x_{n}\right)$ is said to be convergent if there exists $x \in X$ such that $M\left(x_{n}, x, t\right)=1,(\forall) t>0$. In this case, $x$ is called the limit of the sequence $\left(x_{n}\right)$ and we note $\lim _{n \rightarrow \infty} x_{n}=x$, or $x_{n} \rightarrow x$.

Remark 13. Let $(X, M, *, k)$ be a fuzzy b-metric space. A sequence $\left(x_{n}\right)$ is convergent to $x$ if and only if $\left(x_{n}\right)$ is convergent to $x$ in topology $\mathcal{T}_{M}$. 
Indeed,

$$
\begin{gathered}
x_{n} \rightarrow x \text { in topology } \mathcal{T}_{M} \Leftrightarrow \\
\Leftrightarrow(\forall) r \in(0,1),(\forall) t>0,(\exists) n_{0} \in \mathbb{N}: x_{n} \in B(x, r, t),(\forall) n \geq n_{0} \Leftrightarrow \\
\Leftrightarrow(\forall) r \in(0,1),(\forall) t>0,(\exists) n_{0} \in \mathbb{N}: M\left(x_{n}, x, t\right)>1-r,(\forall) n \geq n_{0} \Leftrightarrow \\
\Leftrightarrow \lim _{n \rightarrow \infty} M\left(x_{n}, x, t\right)=1,(\forall) t>0 .
\end{gathered}
$$

Definition 14 . Let $(X, M, *, k)$ be a fuzzy b-metric space and $\left(x_{n}\right)$ be a sequence in $X$. The sequence $\left(x_{n}\right)$ is said to be a Cauchy sequence if

$$
(\forall) r \in(0,1),(\forall) t>0,(\exists) n_{0} \in \mathbb{N}: M\left(x_{n}, x_{m}, t\right)>1-r,(\forall) n, m \geq n_{0} .
$$

A fuzzy b-metric space in which every Cauchy sequence is convergent is called complete fuzzy b-metric space.

\section{$3 \quad$ Fuzzy quasi-b-metric spaces}

Definition 15. A fuzzy quasi-b-metric space is a quadruple $(X, M, *, k)$, where $X$ is a nonempty set, $*$ is a continuous t-norm, $k \geq 1$ is a given real number and $M$ is a fuzzy set in $X \times X \times[0, \infty)$ such that for all $x, y, z \in X$ we have:

(qbM1) $M(x, y, 0)=0$;

(qbM2) $[M(x, y, t)=M(y, x, t)=1,(\forall) t>0]$ if and only if $x=y$;

(qbM3) $M(x, z, k(t+s)) \geq M(x, y, t) * M(y, z, s),(\forall) t, s \geq 0$

(qbM4) $M(x, y, \cdot):[0, \infty) \rightarrow[0,1]$ is left continuous and $\lim _{t \rightarrow \infty} M(x, y, t)=1$.

Remark 16. V. Gregori and S. Romaguera [13] also gave this definition in the particular case $k=1$ and the triple $(X, M, *)$ is called fuzzy quasi-metric space.

Proposition 17. If $Q$ is a fuzzy quasi-b-metric, then $Q^{-1}$ defined by $Q^{-1}(x, y, t)=Q(y, x, t)$ is also a fuzzy quasi-b-metric (called the conjugate of $Q$ ).

Proof: We have to check only $(q b M 3)$.

$$
Q^{-1}(x, z, k(t+s))=Q(z, x, k(s+t)) \geq Q(z, y, s) * Q(y, x, t)=Q^{-1}(x, y, t) * Q^{-1}(y, z, s) .
$$

Definition 18. [18]. Let $*$, $\circ$ be two t-norms. We say that $\circ$ dominates $*$ and we denote $\circ \gg *$ if

$$
\left(x_{1} \circ x_{2}\right) *\left(y_{1} \circ y_{2}\right) \leq\left(x_{1} * y_{1}\right) \circ\left(x_{2} * y_{2}\right),(\forall) x_{1}, x_{2}, y_{1}, y_{2} \in[0,1] \text {. }
$$

Remark 19. [18]. For any t-norm $*$ we have $\wedge \gg *$.

Proposition 20. Let $(X, Q, *, k)$ be a fuzzy quasi-b-metric space and $\circ$ be a continuous t-norm such that $\circ \gg *$. Let $M$ be a fuzzy set in $X \times X \times[0, \infty)$ defined by

$$
M(x, y, t)=Q(x, y, t) \circ Q^{-1}(x, y, t) .
$$

Then $(X, M, *, k)$ is a fuzzy b-metric space. 
Proof: It is easy to check $(b M 1)-(b M 3)$ and $(b M 5)$. We prove $(b M 4)$.

$$
\begin{gathered}
M(x, z, k(t+s))=Q(x, z, k(t+s)) \circ Q^{-1}(x, z, k(t+s)) \geq \\
\geq[Q(x, y, t) * Q(y, z, s)] \circ\left[Q^{-1}(x, y, t) * Q^{-1}(y, z, s)\right] \geq \\
\geq\left[Q(x, y, t) \circ Q^{-1}(x, y, t)\right] *\left[Q(y, z, s) \circ Q^{-1}(y, z, s)\right]=M(x, y, t) * M(y, z, s) .
\end{gathered}
$$

Corollary 21. Let $(X, Q, *, k)$ be a fuzzy quasi-b-metric space and

$$
M(x, y, t)=\min \{Q(x, y, t), Q(y, x, t)\} .
$$

Then $(X, M, *, k)$ is a fuzzy b-metric space.

Proof: We apply previous proposition for $\circ=\wedge \gg *$.

Example 22. Let $(X, d, k)$ be a quasi-b-metric space. Let

$$
M_{d}: X \times X \times[0, \infty) \rightarrow[0,1], M_{d}(x, y, t)=\left\{\begin{array}{rll}
\frac{t}{t+d(x, y)} & \text { if } t>0 \\
0 & \text { if } t=0
\end{array} .\right.
$$

Then $\left(X, M_{d}, \wedge, k\right)$ is a fuzzy quasi-b-metric space. $M_{d}$ will be called standard fuzzy quasi-bmetric.

Proof: The proof is standard.

Proposition 23. If $(X, M, *, k)$ is a fuzzy quasi-b-metric space, then the relation $\leq_{M}$ on $X$ defined by

$$
x \leq_{M} y \text { if and only if } M(x, y, t)=1,(\forall) t>0
$$

is a partial ordering.

Proof. It is easy to check.

\section{$4 \quad$ Fuzzy quasi-pseudo-b-metric spaces}

Definition 24. Let $X$ be a nonempty set. A function $d: X \times X \rightarrow[0, \infty)$ is called quasi-pseudob-metric with constant $k \geq 1$ if the following conditions are satisfied:

$(\mathbf{q p b 1}) d(x, x)=0$

(qpb3) $d(x, z) \leq k[d(x, y)+d(y, z)],(\forall) x, y, z \in X$.

The triple $(X, d, k)$ will be called quasi-pseudo-b-metric space.

Definition 25. A fuzzy quasi-pseudo-b-metric space is a quadruple $(X, M, *, k)$, where $X$ is a nonempty set, $*$ is a continuous t-norm, $k \geq 1$ is a given real number and $M$ is a fuzzy set in $X \times X \times[0, \infty)$ such that for all $x, y, z \in X$ we have:

(qpbM1) $M(x, y, 0)=0$;

(qpbM2) $[M(x, x, t)=1,(\forall) t>0]$;

(qpbM3) $M(x, z, k(t+s)) \geq M(x, y, t) * M(y, z, s),(\forall) t, s \geq 0$; 
(qpbM4) $M(x, y, \cdot):[0, \infty) \rightarrow[0,1]$ is left continuous and $\lim _{t \rightarrow \infty} M(x, y, t)=1$.

Theorem 4.1. Let $(X, M, \wedge, k)$ be a fuzzy quasi-pseudo-b-metric space and

$$
d_{\alpha}(x, y):=\inf \{t>0: M(x, y, t)>\alpha\}, \alpha \in(0,1) .
$$

Then $\mathcal{D}=\left\{d_{\alpha}\right\}_{\alpha \in(0,1)}$ is an ascending family of quasi-pseudo-b-metrics on $X$.

Proof: $(q p 1) d_{\alpha}(x, x)=\inf \{t>0: M(x, x, t)>\alpha\}=0$.

$(q p 2)$

$$
\begin{gathered}
k\left[d_{\alpha}(x, y)+d_{\alpha}(y, z)\right]=k[\inf \{t>0: M(x, y, t)>\alpha\}+\inf \{s>0: M(y, z, s)>\alpha\}]= \\
=k[\inf \{t+s>0: M(x, y, t)>\alpha, M(y, z, s)>\alpha\}]= \\
=\inf \{k(t+s)>0: M(x, y, t) \wedge M(y, z, s)>\alpha\} \geq \\
\geq \inf \{k(t+s)>0: M(x, z, k(t+s))>\alpha\}=d_{\alpha}(x, z) .
\end{gathered}
$$

It remains to prove that $\mathcal{D}=\left\{d_{\alpha}\right\}_{\alpha \in(0,1)}$ is an ascending family. Let $\alpha_{1} \leq \alpha_{2}$. Then

$$
\left\{t>0: M(x, y, t)>\alpha_{2}\right\} \subseteq\left\{t>0: M(x, y, t)>\alpha_{1}\right\} .
$$

Thus

$$
\inf \left\{t>0: M(x, y, t)>\alpha_{2}\right\} \geq \inf \left\{t>0: M(x, y, t)>\alpha_{1}\right\},
$$

namely $d_{\alpha_{2}}(x, y) \geq d_{\alpha_{1}}(x, y),(\forall)(x, y) \in X \times X$.

\section{Conclusions and further works}

In this paper we introduce the notions of fuzzy b-metric space and fuzzy quasi-b-metric space. Thus, we have built a fertile ground to study, in further papers, some fixed point theorems in these spaces. The first problem is to established fuzzy versions of Banach contraction mapping principle in fuzzy b-metric spaces. From here we will obtain a lot of applications both in Mathematics as well as in Engineering and Computer Science. The second issue is to study set-valued contractions in fuzzy b-metric spaces and their applications in control theory and convex optimization. A real challenge is to extend the results of C. Chifu and G. Petrussel [9] in fuzzy b-metric spaces. We intend to obtain some fixed point theorems for multivalued operators in fuzzy b-metric spaces endowed with a graph. This paper may be of interest for researchers working in the following fields belonging to Computer Science and Information Technology:

(i) Integrated solution in computer-based control and communications

(ii) Computational intelligence methods

(iii) Advanced decision support systems

where fuzzy metric spaces will be applied in dealing with the problems such as: fixed point theorems and their applications in the semantics of programs; distance measurement between programs with important results to measure the complexity of programs and algorithms; color image processing and image denoising; the use of some types of fuzzy metrics in cognitive information, in time series and in bioinformatics; the appplications in neural networks; data mining and web mining applications. 


\section{Bibliography}

[1] Alghamdi, M.A., Hussain, N., Salimi, P. (2013); Fixed point and coupled fixed point theorems on b-metric-like spaces, Journal of Inequalities and Applications, 2013:402.

[2] Amini-Harandi, A. (2012). Metric-like spaces, partial metric spaces and fixed points, Fixed Point Theory and Applications, 2012:204.

[3] Bag, T. (2013); Fuzzy cone metric spaces and fixed point theorems of contractive mappings, Annals of Fuzzy Mathematics and Informatics, 6(3): 657-668.

[4] Bag, T. (2014); Some fixed point theorems in fuzzy cone b-metric spaces, International Journal of Fuzzy Mathematics and Systems, 4(2): 255-267.

[5] Bakhtin, I.A. (1989); The contraction mapping principle in quasi-metric spaces, Funct. Anal. Unianowsk Gos. Ped. Inst., 30: 26-37.

[6] Boriceanu, M., Bota, M., Petruşel, A. (2010); Multivalued fractals in b-metric spaces, Central European Journal of Mathematics, 8(2): 367-377.

[7] Boriceanu, M., Petruşel, A., Rus, I.A. (2010); Fixed point theorems for some multivalued generalized contraction in b-metric spaces, International J. Math. Statistics, 6: 65-76.

[8] Boriceanu, M. (2009); Strict fixed point theorems for multivalued operators in b-metric spaces, Intern. J. Modern Math., 4: 285-301.

[9] Chifu, C., Petruşel, G. (2014); Fixed point for multivalued contraction in b-metric spaces with applications to fractals, Taiwanese Journal of Mathematics, 18(5): 1365-1375.

[10] Czerwik, S. (1993); Contraction mappings in b-metric space, Acta Math. Inf. Univ. Ostraviensis, 1: 5-11.

[11] Czerwik, S. (1998); Non-linear set-valued contraction mappings in b-metric spaces, Atti. Sem. Math. Fig. Univ. Modena, 46(2): 263-276.

[12] George, A., Veeramani, P. (1994); On some results in fuzzy metric spaces, Fuzzy Sets and Systems, 64: 395-399.

[13] Gregori, V., Romaguera, S. (2004); Fuzzy quasi-metric spaces, Applied General Topology, 5(1): 128-136.

[14] Hussain, N., Shah, M.H. (2011); KKM mappings in cone b-metric spaces, Comput. Math. Appl., 61(4): 1677-1684.

[15] Kaleva, O., Seikkala, S. (1984); On fuzzy metric spaces, Fuzzy Sets and Systems, 12: 215229.

[16] Kramosil, I., Michálek, J. (1975); Fuzzy metric and statistical metric spaces, Kybernetica, 11: $326-334$.

[17] Matthews, S.G. (1994); Partial metric topology, in: Proc. 8th Summer Conference on General Topology and Applications, Ann. New York Acad. Sci., Vol. 728, The New York Academy of Sciences, 183-197.

[18] Nădăban, S. (2015); Fuzzy euclidean normed spaces for data mining applications, International Journal of Computers Communications \&3 Control, 10(1): 70-77. 
[19] Schweizer, B., Sklar, A. (1960); Statistical metric spaces, Pacific J. Math., 10: 314-334.

[20] Shah, M.H., Hussain, N. (2012); Nonlinear contraction in partially ordered quasi b-metric spaces, Commun. Korean Math. Soc., 27(1): 117-128.

[21] Shatanawi, W., Pitea, A., Lazović, R. (2014); Contraction conditions using comparison function on b-metric spaces, Fixed Point Theory and Applications, 2014:135.

[22] Singh, S.L., Prasad, B. (2008); Some coincidence theorems and stability of iterative procedures, Computers and Mathematics with Applications, 55: 2512-2520.

[23] Zadeh, L.A. (1965); Fuzzy Sets, Informations and Control, 8: 338-353. 